\title{
Effect of weak fluid inertia upon Jeffery orbits
}

\author{
J. Einarsson, ${ }^{1}$ F. Candelier, ${ }^{2}$ F. Lundell,${ }^{3}$ J. R. Angilella, ${ }^{4}$ and B. Mehlig ${ }^{1}$ \\ ${ }^{1}$ Department of Physics, Gothenburg University, SE-41296 Gothenburg, Sweden \\ ${ }^{2}$ University of Aix-Marseille, CNRS, IUSTI UMR 7343, 13013 Marseille, Cedex 13, France \\ ${ }^{3}$ KTH Royal Institute of Technology, SE-100 44 Stockholm, Sweden \\ ${ }^{4}$ Department of Mathematics and Mechanics, LUSAC-ESIX 50130 Cherbourg, University of Caen, France
}

(Received 27 January 2015; published 28 April 2015)

\begin{abstract}
We consider the rotation of small neutrally buoyant axisymmetric particles in a viscous steady shear flow. When inertial effects are negligible the problem exhibits infinitely many periodic solutions, the "Jeffery orbits." We compute how inertial effects lift their degeneracy by perturbatively solving the coupled particle-flow equations. We obtain an equation of motion valid at small shear Reynolds numbers, for spheroidal particles with arbitrary aspect ratios. We analyze how the linear stability of the "log-rolling" orbit depends on particle shape and find it to be unstable for prolate spheroids. This resolves a puzzle in the interpretation of direct numerical simulations of the problem. In general, both unsteady and nonlinear terms in the Navier-Stokes equations are important.
\end{abstract}

DOI: 10.1103/PhysRevE.91.041002

PACS number(s): 47.55.Kf, 47.10.-g, 47.15.G-, 83.10.Pp

Consider a small neutrally buoyant axisymmetric particle rotating in a steady viscous shear flow. This problem was solved by Jeffery [1]. He found that the particle tumbles periodically: it aligns with the flow direction for a long time and then rapidly changes orientation by 180 degrees. There are infinitely many marginally stable periodic orbits, the "Jeffery orbits." This degeneracy means that small perturbations may have substantial consequences. It is thus necessary to consider perturbations due to physical effects neglected in Jeffery's theory.

For very small particles rotational diffusion must be taken into account [2]. The resulting orientational dynamics forms the basis for the theoretical understanding of the rheology of dilute suspensions [3,4]. A second important perturbation is breaking of axisymmetry. It is known that the rotation of small particles in a simple shear depends very sensitively on their shape [5-7]. Third, for larger particles inertial effects must become important. This is the question we address here. To compute the effect of particle inertia is straightforward $[8,9]$. But to determine the effect of fluid inertia on the tumbling is much more difficult. Despite the significance of the question there are few theoretical results, we discuss them in connection with our results below.

To understand the effect of fluid inertia on the motion of particles suspended in a fluid is a question of fundamental importance. But in general it is impractical to solve the coupled particle-flow problem, and there is a long history of deriving approximate equations of motion for the particles, taking into account the unsteady and nonlinear convective terms in the Navier-Stokes equations [10]. The translational motion of a sphere in nonuniform flows at low Reynolds numbers, for example, is approximately described allowing for unsteadiness of the disturbance flow but neglecting convective fluid inertia $[11,12]$. There are many examples where convective

Published by the American Physical Society under the terms of the Creative Commons Attribution 3.0 License. Further distribution of this work must maintain attribution to the author(s) and the published article's title, journal citation, and DOI. fluid inertia must be taken into account, leading to drag and lift effects [13-16]. In most cases either the unsteady or the nonlinear term in the Navier-Stokes equations are considered (but see Refs. [17] and [18]). In our problem both unsteady and nonlinear convective effects matter.

We have derived an equation of motion for the orientation of a neutrally buoyant spheroid in a steady shear when inertial effects are weak but essential. We show how the unsteady and convective terms in the Navier-Stokes equations determine the dynamics. Our results explain how the degeneracy of the Jeffery orbits is lifted by weak inertia. We concentrate on four examples that have been discussed in the literature [19-24]: tumbling and log rolling of prolate and oblate particles (Fig. 1).

In this Rapid Communication we give only a brief account of the formulation of the problem and its perturbative solution (Secs. 1 and 2). We focus on the main results, Eqs. (6), (7), and (8), and explain their implications. Details of our calculation are given in Ref. [25].

1. Formulation of the problem. Tumbling of a spheroid in a simple shear is governed by the shear Reynolds number $\operatorname{Re}_{\mathrm{s}}=$ $s a^{2} \rho_{\mathrm{f}} / \mu$ (fluid inertia), the Stokes number $\mathrm{St}=\left(\rho_{\mathrm{p}} / \rho_{\mathrm{f}}\right) \operatorname{Re}_{\mathrm{s}}$ (particle inertia), and the particle aspect ratio $\lambda$. Here $s$ denotes the shear rate, $\rho_{\mathrm{f}}$ and $\rho_{\mathrm{p}}$ are fluid- and particle-mass densities, and $\mu$ is the dynamic viscosity of the fluid. We reserve $a$ for the major axis length of the particle (used in the definitions of $\operatorname{Re}_{\mathrm{s}}$ and $\mathrm{St}$ ). The aspect ratio is defined as the ratio of lengths along and perpendicular to the symmetry axis. Thus, $\lambda=a / b>1$ (prolate particle) and $\lambda=b / a<1$ (oblate particle), where $b$ is the minor particle-axis length. We dedimensionalize the problem by using the inverse shear rate $s^{-1}$ as time scale, particle size $a$ as length scale, and $\mu s$ as pressure scale. For a neutrally buoyant particle $\mathrm{Re}_{\mathrm{s}}=\mathrm{St}$. To distinguish the contributions from particle and fluid inertia we keep these two parameters separate. In dimensionless variables the angular equations of motion for an axisymmetric particle read

$$
\begin{aligned}
\dot{\boldsymbol{n}} & =\boldsymbol{\omega} \times \boldsymbol{n}, \\
\mathrm{St} \dot{\boldsymbol{L}} & =\operatorname{St}(\mathbb{I} \dot{\boldsymbol{\omega}}+\dot{\mathbb{I}} \boldsymbol{\omega})=\boldsymbol{T} .
\end{aligned}
$$

Here $\boldsymbol{n}$ is the unit vector along the particle symmetry axis. Dots denote time derivatives, $\boldsymbol{L}$ is the particle angular 

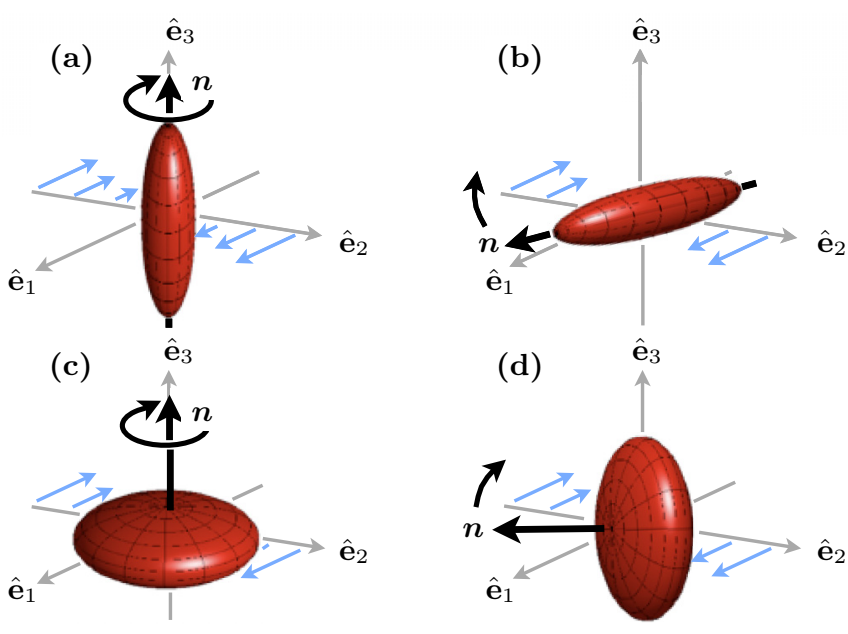

FIG. 1. (Color online) Spheroid in a simple shear. The flow direction is $\hat{\mathbf{e}}_{1}$, shear direction $\hat{\mathbf{e}}_{2}$, and the vorticity points in the negative $\hat{\mathbf{e}}_{3}$-direction. (a) Log-rolling of a prolate particle, $\boldsymbol{n}$ is a unit vector along the symmetry axis of the particle. (b) Tumbling in the shear plane of a prolate particle. (c) Log-rolling of an oblate particle. (d) Tumbling of an oblate particle.

momentum, $\mathbb{I}$ is the moment-of-inertia matrix of the particle. The particle angular velocity is $\boldsymbol{\omega}$, and $\boldsymbol{T}$ is the torque that the fluid exerts on the particle. To find the torque one must solve the Navier-Stokes equations for the flow velocitiy $\boldsymbol{u}$ and pressure $p$ subject to no-slip boundary conditions on the particle surface $\mathscr{S}$ :

$$
\begin{aligned}
& \operatorname{Re}_{\mathrm{s}}\left(\partial_{t} \boldsymbol{u}+\boldsymbol{u} \cdot \nabla \boldsymbol{u}\right)=-\nabla p+\nabla^{2} \boldsymbol{u}, \quad \nabla \cdot \boldsymbol{u}=0, \\
& \boldsymbol{u}=\boldsymbol{\omega} \times \boldsymbol{r} \text { for } \boldsymbol{r} \in \mathscr{S}, \text { and } \boldsymbol{u}=\boldsymbol{u}^{\infty} \text { as }|\boldsymbol{r}| \rightarrow \infty
\end{aligned}
$$

Here $\boldsymbol{r}$ is a spatial coordinate vector with components $\left(r_{1}, r_{2}, r_{3}\right)$ in the Cartesian coordinate system $\hat{\mathbf{e}}_{1}, \hat{\mathbf{e}}_{2}, \hat{\mathbf{e}}_{3}$ shown in Fig. 1. The undisturbed flow field, $\boldsymbol{u}^{\infty}$, is a simple shear flow. We write it as $\boldsymbol{u}^{\infty}=r_{2} \hat{\mathbf{e}}_{1}$, so that its gradient matrix $\mathbb{A}$ has only one nonzero element, $A_{i j}=\delta_{i 1} \delta_{j 2}$. We decompose $\mathbb{A}$ into its symmetric part $\mathbb{S}=\left(\mathbb{A}+\mathbb{A}^{\mathrm{T}}\right) / 2$, and its antisymmetric part $\mathbb{O}=\left(\mathbb{A}-\mathbb{A}^{\mathrm{T}}\right) / 2$.

2. Perturbation theory. The hydrodynamic torque in Eq. (1b) derives from the solutions of Eq. (2). The boundary conditions Eq. (2b) in turn depend on both particle orientation $\boldsymbol{n}$ and particle angular velocity $\boldsymbol{\omega}$. Thus, Eqs. (1) and (2) are coupled and present a difficult problem. To proceed we use a reciprocal theorem $[17,20,26]$ to calculate the torque. Following Ref. [17] we find for the particular case of a simple shear flow:

$$
\boldsymbol{T}=\boldsymbol{T}^{(0)}-\operatorname{Re}_{\mathrm{s}} \int_{\mathscr{V}} d v \widetilde{\mathbb{U}}(\underbrace{\partial_{t} \boldsymbol{u}}_{\begin{array}{c}
\text { unsteady } \\
\text { fluid inertia }
\end{array}}+\underbrace{\boldsymbol{u} \cdot \nabla \boldsymbol{u}}_{\begin{array}{c}
\text { convective } \\
\text { fluid inertia }
\end{array}}) .
$$

The first term $\boldsymbol{T}^{(0)}$ in Eq. (3) is the viscous torque computed by Jeffery [1]. The volume integral is the $O\left(\mathrm{Re}_{\mathrm{s}}\right)$-correction to the hydrodynamic torque. The integral is taken over the entire fluid volume $\mathscr{V}$ outside the particle. The elements of the matrix $\widetilde{U}$ are obtained by solving an auxiliary Stokes problem. Details are given in Ref. [25].

Equation (3) is exact. The difficulty is that the integrand depends on the sought solution $\boldsymbol{u}$ of Eq. (2). Therefore, we follow Refs. [17] and [20] and evaluate Eq. (3) to order $O\left(\mathrm{Re}_{\mathrm{s}}\right)$, the integrand is then only needed to $O(1)$. More precisely, we assume that $\mathrm{St}$ and $\mathrm{Re}_{\mathrm{s}}$ are small and of the same order, so that $\mathrm{Re}_{\mathrm{s}} \mathrm{St}$ is negligible. This allows us to use the known $\mathrm{Re}_{\mathrm{s}}=\mathrm{St}=0$ solutions of Eq. (2) in Eq. (3). The two terms in the integrand in Eq. (3) have the interpretations given in the equation, to linear order in $\mathrm{Re}_{\mathrm{s}}$.

To obtain an equation of motion for $\boldsymbol{n}$ we substitute the hydrodynamic torque Eq. (3) into Eq. (1b) and expand:

$$
\omega=\omega^{(0)}+\operatorname{St} \omega^{(\mathrm{St})}+\operatorname{Re}_{\mathrm{s}} \omega^{\left(\mathrm{Re}_{\mathrm{s}}\right)}+\ldots
$$

Each order in St and $\mathrm{Re}_{\mathrm{s}}$ must satisfy Eqs. (1b) and (3), determining the contributions on the right-hand side of Eq. (4). To lowest order we find the condition $\boldsymbol{T}^{(0)}=0$. It gives

$$
\boldsymbol{\omega}^{(0)}=\boldsymbol{\Omega}+\Lambda \boldsymbol{n} \times \mathbb{S} \boldsymbol{n},
$$

where $\Lambda=\left(\lambda^{2}-1\right) /\left(\lambda^{2}+1\right)$ and $\boldsymbol{\Omega}=\left(\nabla \times \boldsymbol{u}^{\infty}\right) / 2$, so that $\mathbb{O} \boldsymbol{n}=\boldsymbol{\Omega} \times \boldsymbol{n}$. Equation (5) is Jeffery's result [1] for the angular velocity of a spheroid in a simple shear, in the absence of inertial effects. The second term in Eq. (4), the St correction, is found to be equivalent to a result given by Einarsson et al. [9]. We do not reproduce the details here because the expression for $\omega^{(\mathrm{St})}$ is lengthy. The third term, the $O\left(\mathrm{Re}_{\mathrm{s}}\right)$ correction, involves the integral in Eq. (3). But even in perturbation theory [evaluating the integrand to order $O(1)$ ] it is difficult to perform the integral for arbitrary orientations $\boldsymbol{n}$.

3. Symmetries. Exploiting the symmetries of the problem we can show that it is enough to evaluate the integral for only four directions $\boldsymbol{n}$. The corresponding four integrals suffice to determine the orientational equation of motion for $\boldsymbol{n}$. Here we discuss the idea and give the resulting equation of motion. Details are found in Ref. [25].

The small-St and $-\mathrm{Re}_{\mathrm{s}}$ corrections to Jeffery's equation of motion are quadratic in $\mathbb{A}=\mathbb{O}+\mathbb{S}$. The symmetries listed in Table I constrain the form of these contributions. The resulting equation of motion has only four degrees of freedom, which we denote $\beta_{1}, \ldots, \beta_{4}$ :

$$
\begin{aligned}
\dot{\boldsymbol{n}}= & \mathbb{O} \boldsymbol{n}+\Lambda[\mathbb{S} \boldsymbol{n}-(\boldsymbol{n} \cdot \mathbb{S} \boldsymbol{n}) \boldsymbol{n}] \\
& +\beta_{1}(\boldsymbol{n} \cdot \mathbb{S} \boldsymbol{n}) \mathbb{P} \boldsymbol{S} \boldsymbol{n}+\beta_{2}(\boldsymbol{n} \cdot \mathbb{S} \boldsymbol{n}) \mathbb{O} \boldsymbol{n} \\
& +\beta_{3} \mathbb{P} \mathbb{O} \boldsymbol{S} \boldsymbol{n}+\beta_{4} \mathbb{P} \mathbb{S} \boldsymbol{S} .
\end{aligned}
$$

The right-hand side of the first row is Jeffery's equation, it follows from Eqs. (1a) and (5). The remaining terms are all the terms quadratic in $\mathbb{A}=\mathbb{O}+\mathbb{S}$ that are allowed by the symmetries listed in Table $\mathrm{I}$. The projection $\mathbb{P}$ projects out components in the $\boldsymbol{n}$ direction: $\mathbb{P} \boldsymbol{x}=\boldsymbol{x}-(\boldsymbol{n} \cdot \boldsymbol{x}) \boldsymbol{n}$. The four

TABLE I. Symmetries constraining the form of Eq. (6).

Incompressibility:

Symmetry of $\mathbb{S}$ :

Antisymmetry of $\mathbb{O}$ :

Steady shear:

Normalization of $\boldsymbol{n}$ :

Inversion symmetry:

$$
\begin{gathered}
\operatorname{Tr} \mathbb{S}=0 \\
\mathbb{S}^{\mathrm{T}}=\mathbb{S} \\
\mathbb{O}^{\mathrm{T}}=-\mathbb{O} \\
\mathbb{O} \mathbb{O}=-\mathbb{S} \mathbb{S}, \mathbb{O}=-\mathbb{S} \mathbb{O} \\
\boldsymbol{n} \cdot \dot{\boldsymbol{n}}=0
\end{gathered}
$$

invariance under $\boldsymbol{n} \rightarrow-\boldsymbol{n}, \dot{\boldsymbol{n}} \rightarrow-\dot{\boldsymbol{n}}$ 
scalar coefficients $\beta_{\alpha}$ are linear in $\mathrm{St}$ and $\mathrm{Re}_{\mathrm{s}}$, and functions of the particle aspect ratio: $\beta_{\alpha}=\operatorname{St} \beta_{\alpha}^{(\mathrm{St})}(\lambda)+\operatorname{Re}_{\mathrm{s}} \beta_{\alpha}^{\left(\mathrm{Re}_{\mathrm{s}}\right)}(\lambda)$. To obtain these functions we evaluate Eq. (4) directly for four suitably chosen directions $\boldsymbol{n}$. Comparison with Eq. (6) gives a linear system of equations that can be solved for the $\beta_{\alpha}$.

4. Results for the coefficients $\beta_{\alpha}$. In two important limiting cases, the integrand in Eq. (3) simplifies so that we can derive explicit formulas for the coefficients $\beta_{\alpha}$. Details are given in Ref. [25].

First, in the limit of large aspect ratios we find that particle inertia does not contribute, $\beta_{\alpha}^{(\mathrm{St})}(\lambda)=0$, and we obtain that the $\beta_{\alpha}$ coefficients are asymptotic to

$$
\beta_{1}=\frac{7 \operatorname{Re}_{\mathrm{s}}}{30 \log (2 \lambda)-45}, \quad \beta_{2}=\frac{3 \beta_{1}}{7}, \quad \beta_{3}=\beta_{4}=0,
$$

for large values of $\lambda$. The large- $\lambda$ asymptote of Eq. (7) agrees with the slender-body limit obtained in Ref. [20], up to a factor of $8 \pi$. We cannot explain this factor but have verified our results by comparing with an independent calculation (Ref. [27], see below).

Second, we can evaluate the limit of nearly spherical particles. We set $\lambda=1 /(1-\epsilon)$ and find to $O(\epsilon)$ :

$$
\begin{aligned}
& \beta_{1}=0, \quad \beta_{2}=\epsilon\left(\mathrm{St} / 15+\operatorname{Re}_{\mathrm{s}} / 35\right), \\
& \beta_{3}=\epsilon\left(\mathrm{St} / 15-37 \mathrm{Re}_{\mathrm{s}} / 105\right), \quad \beta_{4}=\epsilon\left(\mathrm{St} / 15+11 \mathrm{Re}_{\mathrm{s}} / 35\right) .
\end{aligned}
$$

In this case particle inertia contributes, and this contribution is consistent with the results of Ref. [9] and also with Eqs. (3.15) and (3.16) in Ref. [21].

But the correction due to fluid inertia differs from the earlier results, Eq. (7) in Ref. [19] and Eq. (4.22) in Ref. [21]. In Ref. [19], the Navier-Stokes equations (2) were solved iteratively with approximate boundary conditions. Only the final result is given, thus we cannot determine whether the problem lies in the method or in the algebra. We note that Saffman's assertion that particle inertia can be neglected is incorrect, as Eq. (8) and the results of Ref. [9] show. We have also verified Eq. (8) by an independent calculation, based on a joint perturbation theory in $\epsilon$ and $\mathrm{Re}_{\mathrm{s}}$ using a basis expansion in spherical harmonics. The results are summarized in Ref. [27] and agree with Eq. (8). We also note that Eq. (4.22) of Ref. [21] violates the particle inversion symmetry (Table I).

It follows from Eq. (3) that the unsteady and convective fluid-inertia terms contribute linearly to $\beta_{\alpha}^{\left(\mathrm{Re}_{s}\right)}$. This enables us to separate their effects to order $\mathrm{Re}_{\mathrm{s}}$. For large values of the aspect ratio $\lambda$ we find that unsteady fluid inertia contributes $(8 \log 2 \lambda-12)^{-1}$ to $\beta_{1}^{\left(\mathrm{Re}_{\mathrm{s}}\right)}$ and $\beta_{2}^{\left(\mathrm{Re}_{\mathrm{s}}\right)}$. Comparison with Eq. (7) shows that the contribution from convective fluid inertia is of the same order. For nearly spherical particles, by contrast, we find that convective inertia dominates (order $\epsilon$ ), while the contribution from unsteady fluid inertia is smaller, of order $\epsilon^{2}$.

5. Angular dynamics and linear stability analysis. The inertial corrections in Eq. (6) are small in magnitude when $\mathrm{Re}_{\mathrm{s}}=\mathrm{St}$ is small, but they are important because they destroy the degeneracy of the Jeffery orbits. We illustrate this effect by analyzing four cases: log-rolling along the vorticity axis and tumbling in the flow-shear plane, for prolate and oblate particles (Fig. 1). In the absence of inertial effects these orbits are neutrally stable, as all Jeffery orbits in this limit.
Our analysis is motivated by the fact that recent direct numerical simulation (DNS) results [22-24] of the problem at small but finite $\mathrm{Re}_{\mathrm{s}}$ have resulted in a debate as to whether log rolling is stable for prolate particles, or not. We rewrite Eq. (6) in spherical coordinates, $n_{1}=\sin \theta \cos \varphi, n_{2}=\sin \theta \sin \varphi$, $n_{3}=\cos \theta$ (the Cartesian coordinates are defined in Fig. 1):

$$
\begin{aligned}
\dot{\varphi} \equiv & f(\varphi, \theta)=(\Lambda \cos 2 \varphi-1) / 2+\left(\beta_{1} / 8\right) \sin ^{2} \theta \sin 4 \varphi \\
& -\sin 2 \varphi\left(\beta_{2} \sin ^{2} \theta+\beta_{3}\right) / 4 \\
\dot{\theta} \equiv & g(\varphi, \theta)=\Lambda \sin \theta \cos \theta \sin \varphi \cos \varphi \\
& +\sin \theta \cos \theta\left(\beta_{1} \sin ^{2} \theta \sin ^{2} 2 \varphi+\beta_{3} \cos 2 \varphi+\beta_{4}\right) / 4
\end{aligned}
$$

Equation (9) admit two equilibria for $\theta, \log$ rolling $(\theta=0)$, and tumbling in the shear plane $(\theta=\pi / 2)$; see Fig. 1 .

Consider first the linear stability of the tumbling orbit. The angle $\varphi$ is a monotonously decreasing function of time for infinitesimal values of $\operatorname{Re}_{\mathrm{s}}=\mathrm{St}$. We can thus parametrize the orbit by $\varphi$ instead of time, noting that $\varphi$ changes from 0 to $-2 \pi$ during the period time $T_{p}=4 \pi / \sqrt{1-\Lambda^{2}}$. We obtain a onedimensional periodically driven dynamical system $d \theta / \mathrm{d} \varphi=$ $g(\varphi, \theta) / f(\varphi, \theta)$. We define the stability exponent as the rate of separation in one period:

$$
\gamma_{\mathrm{T}}=T_{p}^{-1} \lim _{\delta \theta_{0} \rightarrow 0} \log \left|\delta \theta_{-2 \pi} / \delta \theta_{0}\right| .
$$

Here $\delta \theta_{0}$ is a small initial separation from $\pi / 2$ at $\varphi=0$, and $\delta \theta_{-2 \pi}$ is the value of this separation at $\varphi=-2 \pi$, after one period. Linearization of the $\theta$ dynamics gives

$$
\gamma_{\mathrm{T}}=T_{p}^{-1} \int_{0}^{-2 \pi} \mathrm{d} \varphi \frac{\partial}{\partial \theta} \frac{g(\varphi, \pi / 2)}{f(\varphi, \pi / 2)} .
$$

Evaluating the integral Eq. (11) to order $\mathrm{Re}_{\mathrm{s}}$ yields an expression for the exponent $\gamma_{\mathrm{T}}$, linear in $\beta_{\alpha}$ :

$$
\gamma_{\mathrm{T}}=-\frac{\beta_{4}}{4}+\frac{1-\sqrt{1-\Lambda^{2}}}{4 \Lambda^{2}}\left(\Lambda \beta_{2}-\beta_{1}\right) .
$$

Log-rolling is a fixed point of the dynamics Eq. (6), not a periodic orbit. But its stability exponent can be calculated as outlined above since the $\varphi$-dynamics decouples from that of $\theta$; see also Ref. [9]. We find

$$
\gamma_{\mathrm{LR}}=\beta_{4} / 4 \text {. }
$$

Using Eqs. (7) and (8) we obtain in the nearly spherical limit $[\epsilon=(\lambda-1) / \lambda \rightarrow 0]$

$$
\frac{\gamma_{\mathrm{T}}}{\operatorname{Re}_{\mathrm{S}}} \sim-2 \epsilon / 21, \quad \frac{\gamma_{\mathrm{LR}}}{\operatorname{Re}_{\mathrm{S}}} \sim 2 \epsilon / 21
$$

Thus, log-rolling is unstable for nearly spherical prolate particles $(\epsilon>0)$, and tumbling is stable. For nearly spherical oblate particles the stabilities are reversed. An earlier approximate theory by Saffman [19] predicts that log-rolling is stable for neutrally buoyant, near-spherical prolate spheroids at small $\mathrm{Re}_{\mathrm{s}}$; see also Ref. [21]. But stable log rolling has not been observed in DNS for nearly spherical prolate spheroids [2224], and it has been debated how to reconcile this fact with Saffman's prediction. We have corrected Saffman's equation of motion. As Eq. (14) shows, it follows that log-rolling is unstable for prolate spheroids at small $\mathrm{Re}_{\mathrm{s}}$, consistent with the DNS results [23]. 


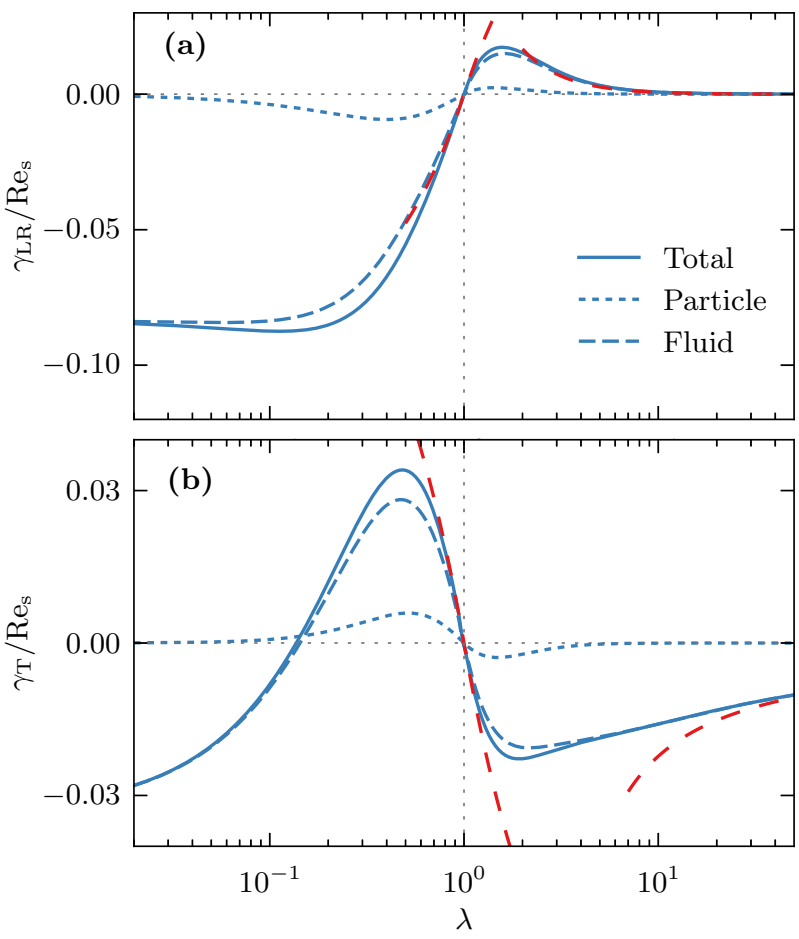

FIG. 2. (Color online) (a) Stability exponent of log-rolling (solid line). Separately shown are particle-inertia (dotted) and fluid-inertia contributions (dashed). Data computed using Eqs. (12) and (13) and numerical results for $\beta_{\alpha}$ (details are given in Ref. [25]). Also shown are the asymptote Eqs. (14) and (15), red dashed lines. (b) Same for tumbling in the shear plane.

In the limit of large aspect ratios we find that the exponents are asymptotic to

$$
\frac{\gamma_{\mathrm{T}}}{\mathrm{Re}_{\mathrm{S}}} \sim(45-30 \log 2 \lambda)^{-1}, \quad \frac{\gamma_{\mathrm{LR}}}{\mathrm{Re}_{\mathrm{S}}} \sim\left(15 \lambda^{2}\right)^{-1}
$$

We see that tumbling is stable in this limit, and log rolling is unstable.

To determine the stability of the tumbling and log-rolling orbits for arbitrary values of $\lambda$ we have computed the $\beta_{\alpha}$ by numerically integrating Eq. (3) for four directions $\boldsymbol{n}$, as outlined above. Figures 2(a) and 2(b) show the resulting exponents. The asymptote Eqs. (14) and (15) are also shown in Fig. 2. Figure 2(a) demonstrates that log rolling is unstable for prolate particles of any aspect ratio. Figures 2(a) and 2(b) also show the separate contributions from fluid and particle inertia to the stability exponents. We see that the contribution of fluid inertia is in general significantly larger than that of particle inertia.

6. Concluding remarks. It would be of great interest to study by DNS how the stability exponents change as $\mathrm{Re}_{\mathrm{s}}$ is increased and to determine how the results described here connect to those of Ref. [24] valid at larger $\mathrm{Re}_{\mathrm{s}}$. Second, we plan to generalize the calculation summarized here to describe wall effects at small $\mathrm{Re}_{\mathrm{s}}$, by the method of reflection [28]. Third, to describe sedimenting particles it is necessary to generalize our results to $\rho_{\mathrm{p}} \neq \rho_{\mathrm{f}}$. Fourth, both the unsteady term and the nonlinear term in the Navier-Stokes equations matter in our problem. This raises the question under which circumstances both effects matter for the tumbling of small particles in unsteady flows, and in particular in turbulence. Finally, we remark that Jeffery orbits are commonly used as benchmarks for DNS, despite being valid only in the limit $\operatorname{Re}_{\mathrm{s}}=0$. Our solutions provide a new reference when fluid inertia is essential but weak.

This work was supported by grants from Vetenskapsrådet and the Göran Gustafsson Foundation for Research in Natural Sciences and Medicine. Support from the MPNS COST Action MP1305 Flowing matter is gratefully acknowledged.
[1] G. B. Jeffery, Proc. R. Soc. A 102, 161 (1922).

[2] E. J. Hinch and L. G. Leal, J. Fluid Mech. 52, 683 (1972).

[3] C. J. Petrie, J. Non-Newton. Fluid 87, 369 (1999).

[4] F. Lundell, D. Soderberg, and H. Alfredsson, Annu. Rev. Fluid Mech. 43, 195 (2011).

[5] E. J. Hinch and L. G. Leal, J. Fluid Mech. 92, 591 (1979).

[6] A. Yarin, O. Gottlieb, and I. Roisman, J. Fluid Mech. 340, 83 (1997).

[7] J. Einarsson, B. Mihiretie, A. Laas, S. Ankardal, J.R. Angilella, D. Hanstorp, and B. Mehlig, arXiv:1503.03023.

[8] F. Lundell and A. Carlsson, Phys. Rev. E 81, 016323 (2010).

[9] J. Einarsson, J. R. Angilella, and B. Mehlig, Physica D 278, 79 (2014).

[10] L. G. Leal, Annu. Rev. Fluid Mech. 12, 435 (1980).

[11] M. R. Maxey and J. J. Riley, Phys. Fluids 26, 883 (1983).

[12] R. Gatignol, J. Méc. Théor. Appl. 2, 143 (1983).

[13] I. Proudman and J. R. A. Pearson, J. Fluid Mech. 2, 237 (1957).

[14] P. G. Saffman, J. Fluid Mech. 22, 385 (1965).
[15] J. McLaughlin, J. Fluid Mech. 224, 261 (1991).

[16] J. Magnaudet, J. Fluid Mech. 485, 115 (2003).

[17] P. Lovalenti and J. Brady, J. Fluid Mech. 256, 561 (1993).

[18] F. Candelier and J.R. Angilella, Phys. Rev. E 73, 047301 (2006).

[19] P. G. Saffman, J. Fluid Mech. 1, 540 (1956).

[20] G. Subramanian and D. L. Koch, J. Fluid Mech. 535, 383 (2005).

[21] G. Subramanian and D. L. Koch, J. Fluid Mech. 557, 257 (2006).

[22] D. Qi and L. Luo, J. Fluid Mech. 477, 201 (2003).

[23] W. Mao and W. Alexeev, J. Fluid Mech. 749, 145 (2014).

[24] T. Rosén, F. Lundell, and C. K. Aidun, J. Fluid Mech. 738, 563 (2014).

[25] J. Einarsson, F. Candelier, F. Lundell, J. R. Angilella, and B. Mehlig, arXiv:1504.02309.

[26] S. Kim, Microhydrodynamics: Principles and Selected Applications (Butterworth-Heinemann, Boston, 1991).

[27] F. Candelier, J. Einarsson, F. Lundell, B. Mehlig, and J. R. Angilella (unpublished).

[28] J. R. Blake and A. T. Chwang, J. Eng. Math. 8, 23 (1974). 